\title{
Aspectos biológicos de Aphis gossypii Glover, 1877 (Hemiptera: Aphididae) em três cultivares de algodoeiro e em três espécies de plantas daninhas
}

\author{
Biological aspects of Aphis gossypii Glover, 1877 (Hemiptera: Aphididae) on three \\ cotton cultivars and on three weeds species
}

Marcos Doniseti Michelotto $^{1}$ Antonio Carlos Busoli $^{2}$

\section{RESUMO}

Este trabalho teve como objetivo estudar os aspectos biológicos de A. gossypii em três cultivares de algodoeiro e em três espécies de plantas daninhas. $O$ experimento foi conduzido no Laboratório de Controle Biológico, sob condições controladas de temperatura $(25 \pm$ $\left.1{ }^{\circ} \mathrm{C}\right)$, umidade relativa do ar $(70 \pm 10 \%)$ e fotofase $(12$ horas). Foram utilizados seis tratamentos, correspondendo às cultivares de algodoeiro CNPA ITA 90, Coodetec 402 e Deltaopal e espécies de plantas daninhas: guanxuma (Sida santaremnensis), malva-preta (Sidastrum micranthum) $e$ trapoeraba (Commelina benghalensis). As unidades experimentais (25 repetições) consistiram de placas de Petri contendo ágar-água a $1 \%$ solidificado. Nestas placas, os ofideos foram mantidos individualmente sobre discos foliares ( $3 \mathrm{~cm}$ de diâmetro) de seus respectivos tratamentos. Os menores valores, em dias, para a fase ninfal foram obtidos em CNPA ITA 90 (4,39 $\pm 0,74)$, Deltaopal $(4,45 \pm 0,75)$, Coodetec $402(4,47 \pm 0,75)$ e trapoeraba $(4,75 \pm 0,80)$. $O$ periodo reprodutivo e a longevidade dos adultos variaram de 8,21 $\pm 0,85$ a 15,52 $\pm 0,93$ e 11,09 $\pm 0,97$ a 24,33 $\pm 1,59$ dias, respectivamente, sobre guanxuma e Deltaopal. As cultivares de algodoeiro são mais favoráveis a A. gossypii que as plantas daninhas e na ausência de plantas de algodoeiro, as plantas daninhas guanxuma, trapoeraba $e$ malva-preta são hospedeiros potenciais para manutenção da população de A. gossypii.

Palavras-chave: pulgão-do-algodoeiro, Gossypium hirsutum, Sida santaremnensis, Sidastrum micranthum, Commelina benghalensis.

\section{ABSTRACT}

The objective of this work was to study the biological aspects of $\boldsymbol{A}$. gossypii on three cotton cultivars and on three weed species. The experiment was carried out in the Laboratory of Biological Control, under controlled conditions of temperature $\left(25 \pm 1{ }^{\circ} \mathrm{C}\right)$, relative humidity $(70 \pm 10 \%)$ and photophase $(12 \mathrm{~h})$. Treatments were: three cotton cultivars (CNPA ITA 90, Coodetec 402 and Deltaopal) and three weed species (Sida santaremnensis, Sidastrum micranthum and Commelina benghalensis). The experimental units (25 replicates) consisted of Petri dishes containing agar-water (1\%) solidified. Aphids were kept individually on leaf discs (3cm diameter) of each respective treatments. The lowest number of days for nymphal phase was obtained on CNPA ITA 90 (4.39 \pm 0.74$)$, Deltaopal $(4.45 \pm$ $0.75)$, Coodetec $402(4.47 \pm 0.75)$ and $\boldsymbol{C}$. benghalensis (4.75 \pm $0.80)$. Reproductive period and adult longevity varied from 8.21 \pm 0.85 to $15.52 \pm 0.93$ and from $11.09 \pm 0.97$ to $24.33 \pm 1.59$ days, respectively on $\boldsymbol{S}$. santaremnensis and Deltaopal. Cotton cultivars are more favorable to $\boldsymbol{A}$. gossypii than weeds and, in the absence of cotton plants, S. santaremnensis, S. micranthum and $\boldsymbol{C}$. benghalensis are potential hosts.

Key words: cotton aphid, Gossypium hirsutum, Sida santaremnensis, Sidastrum micranthum, Commelina benghalensis.

\section{INTRODUÇÃO}

O ofídeo (Aphis gossypii) é espécie cosmopolita (PEÑA-MARTINEZ, 1992), tendo sido

${ }^{1}$ Engenheiro Agrônomo, Pós-graduando em Agronomia (Entomologia Agrícola), Departamento de Fitossanidade, Faculdade de Ciências Agrárias e Veterinárias/UNESP, Via de Acesso Prof. Paulo Donato Castellane, s/nº, 14884-900, Jaboticabal, SP. Bolsista CAPES. Email:mmichelotto@zipmail.com.br Autor para correspondência.

${ }^{2}$ Engenheiro Agrônomo, Professor, Doutor, Departamento de Fitossanidade, Faculdade de Ciências Agrárias e Veterinárias / UNESP. E-mail: acbusoli@fcav.unesp.br 
observado em mais de 90 famílias de plantas (EBERT \& CARTWRIGHT, 1997). Em observações em campo, EKUKOLE (1990) encontrou as maiores populações de $\boldsymbol{A}$. gossypii em algodoeiro (Gossypium hirsutum) e quiabo (Abelmoschus esculentus), e em menores proporções sobre Urena sp. e Sida sp.

No algodoeiro, $\boldsymbol{A}$. gossypii provoca danos diretos pela sucção de seiva e paralisação de crescimento das plantas (COSTA, 1972; GODFREY et al., 2000), provocando também danos indiretos, pela transmissão dos vírus do Vermelhão e do Mosaico das Nervuras forma Ribeirão Bonito (COSTA, 1972; COSTA et al., 1997; FREIRE, 1999). Esta última doença provocou prejuízos de até $1500 \mathrm{~kg} \mathrm{ha}^{-1}$ de algodão em caroço, em municípios de Minas Gerais e Goiás, no ano agrícola de 1997/98 (FREIRE, 1999).

Vários autores estudaram a biologia de $\boldsymbol{A}$. gossypii em hospedeiros, como: abóbora, algodão, quiabo, Urena sp., Sida sp., pepino, melão e crisântemo (KHALIFA \& EL-DIN, 1964; VENDRAMIM \& NAKANO, 1981; EKUKOLE, 1990; ALDYHIM \& KHALIL, 1993; KOCOUREK et al., 1994; STEENIS \& EL-KHAWASS, 1995; KERSTING et al.,1999). Entretanto, com a expansão da cotonicultura para o cerrado brasileiro e a introdução de genótipos de outros países, como Estados Unidos e Austrália, novas cultivares são desenvolvidas e comercializadas todos os anos e não se tem dados sobre a biologia de $\boldsymbol{A}$. gossypii nesses genótipos (FREIRE, 1999).

Esta espécie de ofídeo tem sido encontrada em plantas cultivadas e daninhas, como picão-preto (Bidens pilosa) e guanxumas (Sida sp.) (COSTA, 1972). SANTOS (1999) constatou diversas plantas daninhas como hospedeiros alternativos de $\boldsymbol{A}$. gossypii na Região Centro-Oeste do Brasil, dentre elas a trapoeraba (Commelina benghalensis).

Assim este trabalho teve por objetivo estudar a biologia de $\boldsymbol{A}$. gossypii em três cultivares de algodoeiro (Coodetec 402, CNPA ITA 90 e Deltaopal) e em três espécies de plantas daninhas: trapoeraba e duas malváceas, guanxuma (Sida santaremnensis) e malva-preta (Sidastrum micranthum).

\section{MATERIAL E MÉTODOS}

O experimento foi conduzido no Laboratório de Controle Biológico de Insetos do Departamento de Fitossanidade, Setor de Entomologia da FCAV/UNESP, em Jaboticabal, SP, sendo utilizadas câmaras climatizadas a $25 \pm 1^{\circ} \mathrm{C}$, umidade relativa do ar de $70 \pm 10 \%$ e fotofase de 12 horas.

\section{Criação de manutenção dos ofídeos}

As cultivares de algodoeiro foram semeadas em vasos de polietileno de 5 litros, contendo terra, areia e esterco na proporção 2:1:1 e mantidas em uma gaiola de $2 \times 2 \times 2 \mathrm{~m}$ revestida com tela antiafídeo. As plantas daninhas foram coletadas no campo ainda jovens e transferidas para vasos de 5 litros que continham o mesmo substrato e mantidas na mesma gaiola. Os ofídeos utilizados no experimento foram coletados em colônias em plantas de algodoeiro, no campo, e transferidos para as plantas da gaiola com o auxílio de pincel, para que se alimentassem e se reproduzissem.

\section{Obtenção de ninfas}

Adultos na fase reprodutiva foram coletados nas folhas de cada cultivar de algodoeiro e planta daninha da criação de manutenção e levados até o laboratório. Foram preparados três placas de Petri (6 cm de diâmetro) para cada tratamento. Cada placa conteve $15 \mathrm{ml}$ de solução solidificada de agar-água e um disco foliar de $3 \mathrm{~cm}$ de diâmetro (tratamento), no centro, onde foram criados os adultos que deram origem às ninfas. A tampa de cada placa conteve orifício de $3 \mathrm{~cm}$ de diâmetro, coberto com tela antiafídeo para permitir a aeração e evitar a fuga dos insetos. Em cada placa, foram colocados, com o auxílio de pincel, quinze adultos ápteros de $\boldsymbol{A}$. gossypii e vistoriadas três vezes ao dia para a obtenção das ninfas a serem utilizadas no estudo de biologia.

\section{Condução e avaliação do experimento}

Para cada cultivar e planta daninha, foram utilizadas 25 placas de Petri (repetições), como descrito anteriormente. As folhas foram obtidas no terço superior das plantas, que estavam em casa de vegetação e isentas de produtos fitossanitários. Antes de serem utilizadas, as folhas foram lavadas e posteriormente secas com papel absorvente e, utilizando-se vazador, obtiveramse os discos foliares. Para cada placa, foi transferida uma ninfa com o auxílio de pincel e posteriormente as placas foram tampadas e identificadas. As avaliações foram realizadas duas vezes ao dia e, quando as folhas deterioraram, realizou-se a transferência do ofídio para nova placa contendo solução ágar-água e novo disco foliar.

Avaliou-se a duração da fase ninfal, a duração de cada estádio e as respectivas viabilidades. $\mathrm{Na}$ fase adulta, avaliou-se a duração dos períodos préreprodutivo, reprodutivo e pós-reprodutivo, a longevidade, a produção média diária e total de ninfas produzidas por adulto e o ciclo biológico. Os dados obtidos foram submetidos à análise de variância e as 
médias comparadas pelo teste de Tukey em nível de $5 \%$ de probabilidade de erro.

\section{RESULTADOS E DISCUSSÃO}

\section{Fase ninfal}

Duração dos estádios

A menor duração do primeiro estádio ninfal ocorreu na cultivar Deltaopal (1,07 $\pm 0,07$ dias $)$ e a maior em guanxuma (1,82 $\pm 0,12$ dias) (Tabela 1). Resultados similares foram observados por KHALIFA \& EL-DIN (1964), ao trabalharem com a mesma espécie e hospedeiro, a $27,5^{\circ} \mathrm{C}$, no qual a duração do primeiro estádio foi de $1,07 \pm 0,14$ dias. Os resultados em algodoeiro foram inferiores aos de VENDRAMIM $\&$ NAKANO (1981), que obtiveram 1,60 $\pm 0,05$ dias para ninfas em folhas da cultivar IAC-17.

No segundo estádio ninfal, a maior duração foi verificada nos indivíduos que se alimentaram de malva-preta com 1,53 $\pm 0,12$ dias. Já as ninfas mantidas nas cultivares de algodoeiro não apresentaram diferenças significativas entre si, variando de $1,03 \pm$ 0,07 a $1,16 \pm 0,06$ dias (Tabela 1), valores estes inferiores aos de VENDRAMIM \& NAKANO (1981), que obtiveram $1,35 \pm 0,06$ dias.

O intervalo de variação de duração do terceiro estádio ninfal foi de $0,97 \pm 0,08$ a 1,35 $\pm 0,08$ dias (Tabela 1). As ninfas mantidas em guanxuma apresentaram duração do terceiro estádio maior $(1,35$ $\pm 0,08$ dias) do que àquelas mantidas em folhas de trapoeraba, Deltaopal e Coodetec 402 com, 0,97 $\pm 0,08$,
$1,03 \pm 0,06$ e 1,03 $\pm 0,08$ dias, respectivamente (Tabela 1). Estes dados se assemelham aos de KHALIFA \& ELDIN (1964) e ALDYHIM \& KHALIL (1993), segundo os quais a duração do terceiro estádio foi de $1,08 \pm 0,02 \mathrm{e}$ $1,20 \pm 0,09$ dias, respectivamente, em algodoeiro.

No quarto estádio ninfal, as ninfas que se alimentaram da cultivar CNPA ITA 90 apresentaram período menor com 1,04 $\pm 0,03$ dias, enquanto aquelas que se alimentaram de malva-preta apresentaram período mais longo (1,42 $\pm 0,13$ dias) (Tabela 1$)$. Resultados semelhantes foram observados por KHALIFA \& EL-DIN (1964), para esta espécie, com a duração de 1,29 $\pm 0,16$ dias. Valores superiores foram obtidos por VENDRAMIM \& NAKANO (1981), com $1,58 \pm 0,08$ dias na cultivar IAC-17.

\section{Viabilidade ninfal}

No primeiro estádio, não houve mortalidade nas cultivares de algodoeiro Coodetec 402 e CNPA ITA 90 e em guanxuma. O mesmo ocorreu com indivíduos de segundo estádio, nas cultivares CNPA ITA 90 e Deltaopal e em guanxuma e, no terceiro e quarto estádios, na cultivar Deltaopal e plantas daninhas malva-preta e trapoeraba (Tabela 2).

\section{Duração da fase ninfal}

O intervalo de variação para a fase ninfal foi de 4,39 $\pm 0,74$ a 5,88 $\pm 0,99$ dias (Tabela 1 ), sendo que os maiores períodos foram observados nas ninfas mantidas nas plantas daninhas, malva-preta e guanxuma com $5,51 \pm 0,93$ e $5,88 \pm 0,99$ dias

Tabela 1. Duração média (dias) dos estádios e da fase ninfal de $\boldsymbol{A}$. gossypii em seis plantas. Jaboticabal, SP. 2001.

\begin{tabular}{|c|c|c|c|c|c|c|c|c|c|c|c|}
\hline \multirow{2}{*}{$\begin{array}{l}\text { Plantas } \\
\text { hospedeiras }\end{array}$} & \multirow{2}{*}{ Tratamentos } & \multicolumn{8}{|c|}{ Estádios ninfais } & \multirow{2}{*}{\multicolumn{2}{|c|}{ Fase ninfal }} \\
\hline & & \multicolumn{2}{|l|}{$1^{\mathrm{o}}$} & \multicolumn{2}{|l|}{$2^{\mathrm{o}}$} & \multicolumn{2}{|l|}{$3^{-0}$} & \multicolumn{2}{|l|}{$4^{\mathrm{o}}$} & & \\
\hline \multirow{3}{*}{ Algodoeiro } & Coodetec 402 & $\begin{array}{c}1,11 \pm 0,07^{1} \\
(\mathrm{n}=25)\end{array}$ & $\mathrm{c}^{2}$ & $\begin{array}{c}1,03 \pm 0,07 \\
(\mathrm{n}=24)\end{array}$ & $\mathrm{b}$ & $\begin{array}{c}1,03 \pm 0,08 \\
(\mathrm{n}=24)\end{array}$ & $\mathrm{b}$ & $\begin{array}{c}1,30 \pm 0,08 \\
(n=23)\end{array}$ & $\mathrm{ab}$ & $\begin{array}{c}4,47 \pm 0,75 \\
(n=23)\end{array}$ & $\mathrm{b}$ \\
\hline & $\begin{array}{c}\text { CNPA ITA } \\
90\end{array}$ & $\begin{array}{c}1,18 \pm 0,06 \\
(n=25)\end{array}$ & $\mathrm{bc}$ & $\begin{array}{c}1,05 \pm 0,06 \\
(n=25)\end{array}$ & $\mathrm{b}$ & $\begin{array}{c}1,12 \pm 0,05 \\
(n=24)\end{array}$ & $a b$ & $\begin{array}{c}1,04 \pm 0,03 \\
(n=24)\end{array}$ & $\mathrm{b}$ & $\begin{array}{c}4,39 \pm 0,74 \\
(n=24)\end{array}$ & $\mathrm{b}$ \\
\hline & Deltaopal & $\begin{array}{c}1,07 \pm 0,07 \\
(\mathrm{n}=24)\end{array}$ & $\mathrm{c}$ & $\begin{array}{c}1,16 \pm 0,06 \\
(n=24)\end{array}$ & $a b$ & $\begin{array}{c}1,03 \pm 0,06 \\
(n=24)\end{array}$ & $\mathrm{b}$ & $\begin{array}{c}1,19 \pm 0,05 \\
(n=24)\end{array}$ & $a b$ & $\begin{array}{c}4,45 \pm 0,75 \\
(n=24)\end{array}$ & $\mathrm{b}$ \\
\hline \multirow{3}{*}{ Daninhas } & Guanxuma & $\begin{array}{c}1,82 \pm 0,12 \\
(n=25)\end{array}$ & $\mathrm{a}$ & $\begin{array}{c}1,36 \pm 0,11 \\
(n=25)\end{array}$ & $a b$ & $\begin{array}{c}1,35 \pm 0,08 \\
(n=24)\end{array}$ & $\mathrm{a}$ & $\begin{array}{c}1,35 \pm 0,10 \\
(n=23)\end{array}$ & $a b$ & $\begin{array}{c}5,88 \pm 0,99 \\
(n=23)\end{array}$ & $\mathrm{a}$ \\
\hline & Trapoeraba & $\begin{array}{c}1,49 \pm 0,12 \\
(n=22)\end{array}$ & $\mathrm{ab}$ & $\begin{array}{c}1,05 \pm 0,12 \\
(\mathrm{n}=21)\end{array}$ & $\mathrm{b}$ & $\begin{array}{c}0,97 \pm 0,08 \\
(n=21)\end{array}$ & $\mathrm{b}$ & $\begin{array}{c}1,24 \pm 0,08 \\
(n=21)\end{array}$ & $\mathrm{ab}$ & $\begin{array}{c}4,75 \pm 0,80 \\
(n=21)\end{array}$ & $\mathrm{b}$ \\
\hline & Malva-preta & $\begin{array}{c}1,26 \pm 0,10 \\
(n=22)\end{array}$ & $\mathrm{bc}$ & $\begin{array}{c}1,53 \pm 0,12 \\
(\mathrm{n}=21)\end{array}$ & $\mathrm{a}$ & $\begin{array}{c}1,30 \pm 0,11 \\
(n=21)\end{array}$ & $a b$ & $\begin{array}{c}1,42 \pm 0,13 \\
(n=21)\end{array}$ & $\mathrm{a}$ & $\begin{array}{c}5,51 \pm 0,93 \\
(n=21)\end{array}$ & a \\
\hline Média & & \multicolumn{2}{|c|}{$1,32 \pm 0,04$} & \multicolumn{2}{|c|}{$1,19 \pm 0,04$} & \multicolumn{2}{|c|}{$1,13 \pm 0,03$} & \multicolumn{2}{|c|}{$1,25 \pm 0,04$} & \multicolumn{2}{|c|}{$4,90 \pm 0,08$} \\
\hline CV (\%) & & \multicolumn{2}{|l|}{33,49} & \multicolumn{2}{|l|}{37,40} & \multicolumn{2}{|l|}{32,45} & \multicolumn{2}{|l|}{32,14} & \multicolumn{2}{|l|}{15,10} \\
\hline
\end{tabular}

${ }^{1}$ Média \pm erro padrão. $\mathrm{n}=$ número de indivíduos avaliados.

${ }^{2}$ Médias não seguidas pela mesma letra na coluna diferem significativamente entre si pelo teste de Tukey em nível de $5 \%$ de probabilidade de erro.

Ciência Rural, v. 33, n. 6, nov-dez, 2003. 
Tabela 2 - Viabilidade de ninfas de $\boldsymbol{A}$. gossypii em seis plantas. Jaboticabal, SP. 2001.

\begin{tabular}{|c|c|c|c|c|c|c|}
\hline \multirow{2}{*}{$\begin{array}{l}\text { Plantas } \\
\text { hospedeiras }\end{array}$} & \multirow{2}{*}{ Tratamentos } & \multicolumn{4}{|c|}{ Estádios ninfais } & \multirow{2}{*}{ Fase ninfal } \\
\hline & & $1^{\mathrm{o}}$ & $2^{-}$ & $3^{-}$ & $4^{\mathrm{o}}$ & \\
\hline \multirow{3}{*}{ Algodoeiro } & Coodetec 402 & 100,00 & 96,00 & 100,00 & 95,83 & 92,00 \\
\hline & CNPA ITA 90 & 100,00 & 100,00 & 96,00 & 100,00 & 96,00 \\
\hline & Deltaopal & 96,00 & 100,00 & 100,00 & 100,00 & 96,00 \\
\hline \multirow{3}{*}{ Daninhas } & Guanxuma & 100,00 & 100,00 & 96,00 & 95,83 & 92,00 \\
\hline & Trapoeraba & 88,00 & 95,45 & 100,00 & 100,00 & 84,00 \\
\hline & Malva-preta & 88,00 & 95,45 & 100,00 & 100,00 & 84,00 \\
\hline Média & & 95,33 & 97,82 & 98,67 & 98,61 & 90,67 \\
\hline
\end{tabular}

respectivamente. Ninfas mantidas sobre as cultivares de algodoeiro obtiveram valores variando de 4,39 \pm 0,74 a $4,47 \pm 0,75$ dias (Tabela 1 ). Resultados semelhantes foram obtidos por KHALIFA \& EL-DIN (1964), segundo os quais foi observado duração média de 4,46 $\pm 0,18$ dias para a mesma espécie em algodoeiro, sob temperatura de $27,5^{\circ} \mathrm{C}$.

\section{Fase adulta}

\section{Período pré-reprodutivo}

As maiores durações no período préreprodutivo de adultos ápteros de $\boldsymbol{A}$. gossypii, foram observados em guanxuma, com 0,85 $\pm 0,18$ dias (Tabela 3). ALDYHIM \& KHALIL (1993) e KOCOUREK et al. (1994) observaram períodos superiores em adultos da mesma espécie, quando mantidos em abóbora e pepino, respectivamente, com duração média de 1,0 dia, para ambos.

\section{Período reprodutivo}

Adultos de $\boldsymbol{A}$. gossypii mantidos sobre a cultivar Deltaopal apresentaram maior duração desta fase com 15,52 $\pm 0,93$ dias, do que adultos mantidos sobre guanxuma $(8,21 \pm 0,85$ dias $)$. Nos demais hospedeiros, verificou-se duração intermediária (Tabela 3). De acordo com VENDRAMIM \& NAKANO (1980), o período reprodutivo dos adultos da mesma espécie, mantidos sobre folhas de algodoeiro da cultivar IAC-17, foi superior com 23,20 $\pm 2,34$ dias.

\section{Fecundidade}

As maiores produções de ninfas foram nas cultivares Deltaopal $(5,69 \pm 0,26$ ninfas/adulto/ dia) e Coodetec 402 (5,66 $\pm 0,42$ ninfas/adulto/dia) e na planta daninha trapoeraba $(5,44 \pm 0,31$ ninfas/ adulto/dia) (Tabela 4). Valores inferiores foram observados por VENDRAMIM \& NAKANO (1980) na cultivar IAC-17, segundo os quais adultos de A. gossypii apresentaram fecundidade média de 2,18 ninfas/adulto/dia. Já EKUKOLE (1990) observou que as maiores fecundidades foram em algodoeiro (4,76 ninfas/adulto/dia) e as menores em amendoim (0,64 ninfas/adulto/dia) e Sida $\mathrm{sp}$. (1,44 ninfas/adulto/dia).

Tabela 3 - Duração média (em dias) dos períodos pré-reprodutivo, reprodutivo e pós-reprodutivo e longevidade de $\boldsymbol{A}$. gossypii em seis plantas. Jaboticabal, SP. 2001.

\begin{tabular}{|c|c|c|c|c|c|c|c|c|c|c|}
\hline \multirow{3}{*}{$\begin{array}{l}\text { Plantas } \\
\text { hospedeiras }\end{array}$} & \multirow{3}{*}{$\begin{array}{l}\text { Tratamentos } \\
\text { Coodetec } 402\end{array}$} & \multirow{3}{*}{$\begin{array}{c}\begin{array}{c}\text { Afídeos } \\
\text { avaliados }\end{array} \\
23\end{array}$} & \multicolumn{6}{|c|}{ Períodos } & \multirow{2}{*}{\multicolumn{2}{|c|}{ Longevidade }} \\
\hline & & & \multicolumn{2}{|c|}{ Pré-reprodutivo } & \multicolumn{2}{|c|}{ Reprodutivo } & \multicolumn{2}{|c|}{ Pós-reprodutivo } & & \\
\hline & & & $0,24 \pm 0,07^{1}$ & $\mathrm{~b}^{2}$ & $13,95 \pm 1,08$ & $\mathrm{ab}$ & $7,22 \pm 1,44$ & $a b$ & $21,41 \pm 1,88$ & $a b$ \\
\hline \multirow[t]{3}{*}{ Algodoeiro } & CNPA ITA 90 & 24 & $0,32 \pm 0,09$ & $\mathrm{~b}$ & $13,51 \pm 1,28$ & $a b$ & $3,66 \pm 0,84$ & $\mathrm{bc}$ & $17,49 \pm 1,89$ & $\mathrm{bc}$ \\
\hline & Deltaopal & 24 & $0,21 \pm 0,06$ & $\mathrm{~b}$ & $15,52 \pm 0,93$ & $\mathrm{a}$ & $8,61 \pm 1,36$ & $\mathrm{a}$ & $24,33 \pm 1,59$ & $\mathrm{a}$ \\
\hline & Guanxuma & 23 & $0,85 \pm 0,18$ & $\mathrm{a}$ & $8,21 \pm 0,85$ & $\mathrm{c}$ & $2,09 \pm 0,36$ & $\mathrm{c}$ & $11,09 \pm 0,97$ & $\mathrm{c}$ \\
\hline \multirow[t]{2}{*}{ Daninhas } & Trapoeraba & 21 & $0,47 \pm 0,14$ & $a b$ & $12,11 \pm 0,70$ & $a b c$ & $9,80 \pm 1,13$ & $\mathrm{a}$ & $22,38 \pm 1,23$ & $a b$ \\
\hline & Malva-preta & 21 & $0,45 \pm 0,13$ & $a b$ & $11,01 \pm 0,84$ & $\mathrm{bc}$ & $6,79 \pm 1,43$ & $a b c$ & $18,24 \pm 1,87$ & $\mathrm{ab}$ \\
\hline Média & & & \multicolumn{2}{|c|}{$0,42 \pm 0,05$} & \multicolumn{2}{|c|}{$12,44 \pm 0,44$} & \multicolumn{2}{|c|}{$6,29 \pm 0,52$} & \multicolumn{2}{|c|}{$19,15 \pm 0,75$} \\
\hline CV (\%) & & & \multicolumn{2}{|l|}{28,48} & \multicolumn{2}{|l|}{37,56} & \multicolumn{2}{|l|}{87,22} & \multicolumn{2}{|l|}{40,29} \\
\hline
\end{tabular}

${ }^{1}$ Média \pm erro padrão.

${ }^{2}$ Médias não seguidas pela mesma letra na coluna diferem significativamente entre si pelo teste de Tukey em nível de $5 \%$ de probabilidade de erro. 
Tabela 4 - Produção média diária e total de ninfas por adultos de $\boldsymbol{A}$. gossypii e ciclo biológico, em seis plantas. Jaboticabal, SP. 2001.

\begin{tabular}{|c|c|c|c|c|c|}
\hline \multirow{2}{*}{ Plantas hospedeiras } & \multirow{2}{*}{ Tratamentos } & \multirow{2}{*}{ Afídeos avaliados } & \multicolumn{2}{|c|}{ Fecundidade } & \multirow[t]{2}{*}{ Ciclo biológico (dias) } \\
\hline & & & Ninfas/adulto/dia & Ninfas/adulto & \\
\hline \multirow{3}{*}{ Algodoeiro } & Coodetec 402 & 23 & $5,66 \pm 0,42^{1} \mathrm{a}^{2}$ & $71,96 \pm 4,06 \mathrm{ab}$ & $25,89 \pm 1,88 a b$ \\
\hline & CNPA ITA 90 & 24 & $4,53 \pm 0,28 \mathrm{ab}$ & $58,83 \pm 5,66 \mathrm{bc}$ & $21,87 \pm 1,85 b c$ \\
\hline & Deltaopal & 24 & $5,69 \pm 0,26 \mathrm{a}$ & $84,50 \pm 3,75 \mathrm{a}$ & $28,79 \pm 1,57 \mathrm{a}$ \\
\hline \multirow{3}{*}{ Daninhas } & Guanxuma & 23 & $3,56 \pm 0,25 b$ & $29,70 \pm 3,22 \mathrm{~d}$ & $16,99 \pm 1,01 \mathrm{c}$ \\
\hline & Trapoeraba & 21 & $5,44 \pm 0,31 \mathrm{a}$ & $63,24 \pm 3,34 b$ & $27,15 \pm 1,20 \mathrm{ab}$ \\
\hline & Malva-preta & 21 & $4,13 \pm 0,21 \mathrm{~b}$ & $43,48 \pm 3,34 \mathrm{~cd}$ & $23,75 \pm 1,92 \mathrm{ab}$ \\
\hline Média & & & $4,84 \pm 0,14$ & $58,96 \pm 2,25$ & $24,05 \pm 0,73$ \\
\hline $\mathrm{CV}(\%)$ & & & 29,26 & 32,73 & 32,00 \\
\hline
\end{tabular}

${ }^{1}$ Média \pm erro padrão.

${ }^{2}$ Médias não seguidas pela mesma letra na coluna diferem significativamente entre si pelo teste de Tukey em nível de $5 \%$ de probabilidade de erro.

$\mathrm{Na}$ cultivar Deltaopal, os adultos apresentaram a maior capacidade reprodutiva com 84,50 $\pm 3,75$ ninfas/adulto (Tabela 4). As menores produções de ninfas foram verificadas nos adultos mantidos em guanxuma com 29,70 $\pm 3,22$ ninfas/adulto. De acordo com VENDRAMIM \& NAKANO (1981), a produção de ninfas na cultivar IAC-17 foi de 47,90 \pm 5,06 ninfas/adulto.

\section{Período pós-reprodutivo}

Os maiores períodos foram observados nos adultos mantidos sobre trapoeraba $(9,80 \pm 1,13$ dias) e Deltaopal $(8,61 \pm 1,36)$ e os menores, em adultos mantidos sobre folhas de guanxuma $(2,09 \pm$ 0,36 dias) (Tabela 3 ). Valores próximos aos verificados para a cultivar CNPA ITA 90 (3,66 $\pm 0,84$ dias $)$, foram obtidos por VENDRAMIM \& NAKANO (1981), com 3,60 dias sobre a cultivar IAC-17. STEENIS \& ELKHAWASS (1995) observaram duração de 2,5 dias para o período pós-reprodutivo de adultos de $\boldsymbol{A}$. gossypii, quando mantidos sobre pepino.

\section{Longevidade}

A maior longevidade foi observada em indivíduos mantidos sobre folhas da cultivar Deltaopal com 24,33 $\pm 1,59$ dias, enquanto que a menor longevidade ocorreu em indivíduos mantidos sobre folhas de guanxuma com 11,09 \pm 0,97 dias (Tabela 3). Resultados semelhantes foram observados por KERSTING et al. (1999), em algodoeiro (23,1 dias a $25^{\circ} \mathrm{C}$ ). STEENIS \& EL-KHAWASS (1995) registrou longevidade média de adultos ápteros da mesma espécie, sobre pepino de 14,3 dias, a $25^{\circ} \mathrm{C}$.

\section{Ciclo biológico}

A maior duração do ciclo biológico de $\boldsymbol{A}$. gossypii foi observada nos indivíduos mantidos sobre a cultivar Deltaopal com $28,79 \pm 1,57$ dias e o menor valor nos indivíduos mantidos nas folhas de guanxuma com 16,99 $\pm 1,01$ dias (Tabela 4). ALDYHIM \& KHALIL (1993), trabalhando com a mesma espécie e temperatura, observaram resultados inferiores em plantas de algodoeiro, com ciclo médio de 15,8 dias.

De acordo com EBERT \& CARTWRIGHT (1997), num mesmo hospedeiro, como por exemplo, pepino, diversos autores encontraram diferenças nos resultados obtidos, em função da variabilidade genética existente, que pode ser influenciada pelas diferenças no procedimento experimental. Segundo esses autores, essas diferenças incluem: local onde foi realizado a pesquisa, o tipo de confinamento do afídeo (em discos de folhas, isolamento de toda a planta ou pequenas gaiolas nas folhas), a idade da colônia do afídeo e os limites dos fatores abióticos como luz e temperatura.

Neste trabalho, em iguais condições, observa-se que as cultivares de algodoeiro, quando comparadas às plantas daninhas, são mais favoráveis ao desenvolvimento do afídeo A. gossypii. Em condições de campo, na ausência das plantas de algodoeiro, as plantas daninhas podem servir de hospedeiros alternativos.

\section{CONCLUSÕES}

As cultivares de algodoeiro são mais favoráveis a $\boldsymbol{A}$. gossypii que as plantas daninhas e na ausência de algodoeiro as plantas daninhas guanxuma, trapoeraba e malva-preta são hospedeiros potenciais para manutenção da população de $\boldsymbol{A}$. gossypii.

Ciência Rural, v. 33, n. 6, nov-dez, 2003. 


\section{REFERÊNCIAS BIBLIOGRÁFICAS}

ALDYHIM, Y.N.; KHALIL, A.F. Influence of temperature and daylength on population development of Aphis gossypii on Curcubita pepo. Entomologia Experimentalis et Applicata, Dordrecht, v.67, n.2, p.167-172, 1993.

COSTA, A.S.; JUliATti, F.C.; RUANO, O. Algodão (Gossypium hirsutum L.): Doenças causadas por vírus. In: VALE, F. X. R. do; ZAMBOLIM, L. (Eds). Controle de doenças de plantas: grandes culturas. Viçosa : UFV, 1997. V.2, cap.12, p.571-582.

COSTA, D. O pulgão em evidência. Divulgação Agronômica, São Paulo, v.32, p.19-22, 1972.

EBERT, T.A.; CARTWRIGHT, B. Biology and ecology of Aphis gossypii Glover (Homoptera: Aphididae). Southwestern Entomologist, Dallas, v.22, n.1, p.116-153, 1997.

EKUKOLE, G. Effects of some selected plants on the fecundity of Aphis gossypii Glover under laboratory conditions. Coton et Fibres Tropicales, Paris, v.45, n.3, p.263-266, 1990.

FREIRE, E.C. Doença azul tem solução. Cultivar, Pelotas, v.1, n.1, p.64-65, 1999.

GODFREY, L.D.; ROSENHEIM, J.A.; GOODELL, P.B. Cotton aphid emerges as major pest in SJV cotton. California Agriculture, Oakland, v.54, n.6, p.26-29, 2000.

KHALIFA, A.; EL-DIN, S. Biological and ecological study on Aphis gossypii Glover (Hemiptera: Aphididae). Bulletin
Entomological Society Egypte, Cairo, v.48, p.131-153, 1964.

KERSTING, U.; SATAR, S.; UYGUN, N. Effect of temperature on development rate and fecundity of apterous Aphis gossypii Glover (Homoptera: Aphididae) reared on Gossypium hirsutum L. Journal of Applied Entomology, Dordrecht, v.123, p.23-27, 1999.

KOCOUREK, F. et al. Effects of temperature on development rate and intrinsic rate of increase of Aphis gossypii reared on greenhouse cucumbers. Entomologia Experimental et Applicata, Dordrecht, v.71, n.1, p.5964, 1994.

PEÑA-MARTINEZ, R. Identificación de afidos de importância agricola. In: URIAS-M, C.; RODRÍGUEZ-M, R.; ALEJANDRE-A, T. Afidos como vectores de virus en México. México : Centro de Fitopatologia, Montecillo, 1992. V.2, cap.1. p.1-135.

SANTOS, W.J. Monitoramento e controle das pragas do algodoeiro. In: CIA, E.; FREIRE, E.C.; SANTOS, W.J. (Eds). Cultura do algodoeiro. Piracicaba : Potafós, 1999. Cap.9, p.134-179.

STEENIS, M. J. van; EL-KHAWASS, K.A.M.H. Life history of Aphis gossypii on cucumber: influence of temperature, host plant and parasitism. Entomologia Experimentalis et Applicata, Amsterdam, v.76, p.121-131, 1995.

VENDRAMIM, J.D.; NAKANO, O. Aspectos biológicos de Aphis gossypii Glover, 1877 (Homoptera: Aphididae) em algodoeiro. Anais da Sociedade Entomológica do Brasil, Jaboticabal, v.10, n.2, p.163-173, 1981. 